CERN-TH.7527/94

GEF-TH-9/1994

IFUM $487 / F T$

\title{
Total Cross Sections for Heavy Flavour Production at HERA
}

\author{
Stefano Frixione \\ Dip. di Fisica, Università di Genova, and INFN, Sezione di Genova, Genoa, Italy \\ Michelangelo L. Mangano \\ INFN, Scuola Normale Superiore and Dipartimento di Fisica, Pisa, Italy \\ Paolo Nason $[$ and Giovanni Ridolfi] \\ CERN TH-Division, CH-1211 Geneva 23, Switzerland
}

\begin{abstract}
We compute total cross sections for charm and bottom photoproduction at HERA energies, and discuss the relevant theoretical uncertainties. In particular we discuss the problems arising from the small- $x$ region, the uncertainties in the gluon parton density, and the uncertainties in the hadronic component of the cross section. Total electroproduction cross sections, calculated in the Weizsäcker-Williams approximation, are also given.
\end{abstract}

CERN-TH.7527/94

December 1994

\footnotetext{
${ }^{1}$ Address after Feb. $1^{\text {st }}$ 1995: CERN TH-Division, CH-1211 Geneva 23, Switzerland

${ }^{2}$ On leave of absence from INFN, Sezione di Milano, Milan, Italy.

${ }^{3}$ On leave of absence from INFN, Sezione di Genova, Genoa, Italy.
} 


\section{Introduction}

Photoproduction of heavy flavours has been studied extensively in fixed target experiments (ref. [1]). The measured total cross sections for charm photoproduction turn out to be in reasonable agreement with theoretical expectations (see ref. [2]). At HERA new opportunities are available. On one hand, one can extend in energy previous charm production studies, from center-of-mass energies around $30 \mathrm{GeV}$ to energies up to $300 \mathrm{GeV}$. Some preliminar results have already been presented in ref. [3]. Small- $x$ effects in these high energy regimes may not be negligible for charm production, and it will be interesting to see whether they are important at HERA. Bottom production will also be observable at HERA. The larger value of the bottom quark mass makes QCD predictions more reliable. Furthermore, bottom hadroproduction has been extensively studied at hadron colliders, and the comparison with QCD predictions, although qualitatively good, presents some problems (see refs. [4, 5,6]). Theoretical arguments indicate that perturbation theory should describe photoproduction of heavy flavours more reliably than hadroproduction. This is also supported by experimental evidence in fixed-target charm production. HERA has therefore the opportunity to clarify some open questions in bottom production.

In this paper, we discuss the total cross sections for charm and bottom photoproduction, and for electroproduction in the Weizsäcker-Williams approximation, in the HERA energy regime. Our analysis is based on the next-to-leading order calculation of heavy-quark photoproduction and hadroproduction cross sections performed in refs. [8,9]. Some results in heavy-quark electroproduction at HERA have already been presented in ref. [10]. The computer program we used for this work was developed in refs. [11,12 and is available upon request. We will not consider electroproduction cross sections for large photon virtuality, which has been recently discussed in ref. [7].

Our work is organized as follows. In section 2 we discuss charm photoproduction, focussing especially on the theoretical uncertainties which are specific of HERA physics. In section 3 we give our results for bottom photoproduction. In section 4 we present our results for charm and bottom production in ep collisions, and in section 5 we give our conclusions. 


\section{Charm production}

In charm photoproduction at HERA, because of the large center-of-mass energies available, the new problem arises (which was not present in fixed target configurations) of the presence of a large radiative effect, proportional to $\alpha_{\mathrm{em}} \alpha_{S}^{2} \log \left(S / 4 m_{c}^{2}\right)$, where $S$ is the squared center-of-mass energy of the photon-hadron system, and $m_{c}$ is the charm quark mass. This effect is the first manifestation of a whole tower of corrections behaving like $\alpha_{\mathrm{em}} \alpha_{S}\left(\alpha_{S} \log \left(S / 4 m_{c}^{2}\right)\right)^{n}$, arising from the small- $x$ region. In the high energy limit, $\alpha_{S} \log \left(S / 4 m_{c}^{2}\right)$ may become of order 1 , and one should then worry about resumming all these contributions. A formalism for the resummation has been studied in refs. [13]. In the present work, we will try to assess the importance of these effects at HERA. Following the work of ref. [9], in fig. 1 we show various contributions to the total pointlike photoproduction cross section, histogrammed as a function of the variable $\tau$, defined as the ratio of the partonic versus the hadronic center-of-mass squared energies $\hat{s} / S$.

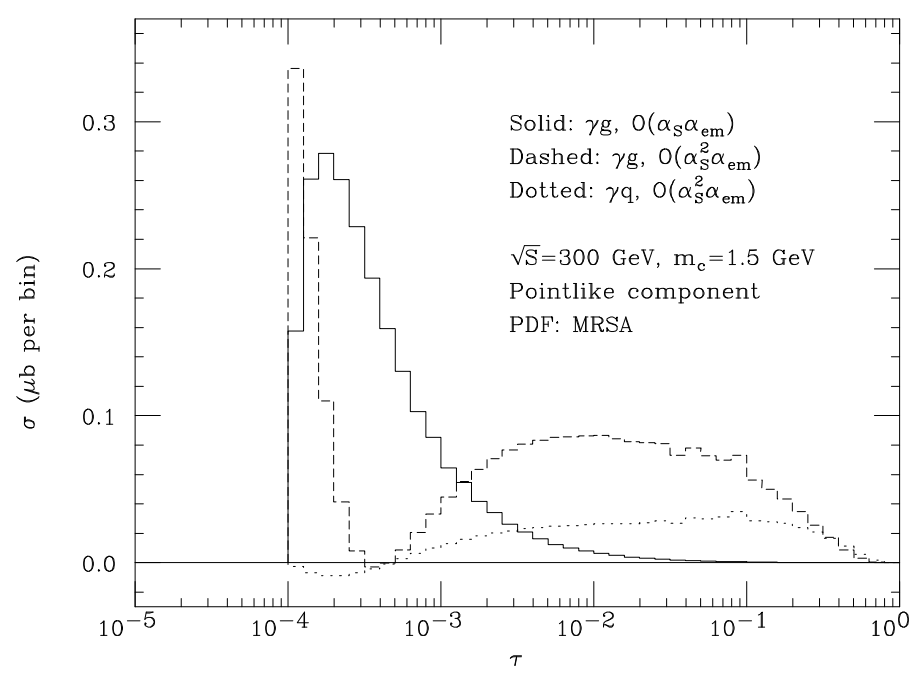

Figure 1: The $\mathcal{O}\left(\alpha_{\mathrm{em}} \alpha_{S}\right) \gamma g, \mathcal{O}\left(\alpha_{\mathrm{em}} \alpha_{S}^{2}\right) \gamma g$ and $\mathcal{O}\left(\alpha_{\mathrm{em}} \alpha_{S}^{2}\right) \gamma q$ contributions to the total charm photoproduction cross section, histogrammed as a function of $\tau=\hat{s} / S$. The proton factorization and renormalization scales have been taken equal to $m_{c}$ in the short-distance cross sections.

In the case of the pointlike component of photoproduction, this ratio is simply 
equal to $x$, the fraction of the hadron momentum carried by the parton. We see that in the next-to-leading $\gamma g$ contribution there is a sizeable hump away from the threshold region. The same behaviour is observed in the $\gamma q$ contribution. The hump arises from the fact that the next-to-leading parton cross section goes to a constant for large $\hat{s}$ (see figs. 2 and 3 of ref. [8]). Therefore, for a hypothetical gluon density of the form $G(x)=1 / x$, the next-to-leading $\gamma g$ contribution to the total cross section would be given by

$$
\int_{4 m_{c}^{2} / s}^{1} \hat{\sigma}_{\gamma g}^{(1)} G(x) d x=\int_{4 m_{c}^{2} / s}^{1} \hat{\sigma}_{\gamma g}^{(1)} d \log x .
$$

Observe that, were it not for the fact that the gluon density has some extra damping factor as $x \rightarrow 1$, the dashed histogram in fig. 11 would go to a constant as $x \rightarrow 1$. In the very high energy limit, the hump will therefore become a plateau, and its width would be roughly equal to $\log \left(S / 4 m_{c}^{2}\right)$. One can show that this problem does not stop at this order: at order $\alpha_{\mathrm{em}} \alpha_{S}^{n}$ the partonic cross section grows like $\left(\log \hat{s} / 4 m_{c}^{2}\right)^{n-1}$, and this would give a contribution to the total cross section raising like $\left(\log \left(S / 4 m_{c}^{2}\right)\right)^{n}$ with the energy. Notice that fig. 11 was obtained using the MRSA parton densities [14, which have a relatively singular gluon distribution at small $x$. This type of parton density emphasizes the threshold region. We therefore expect that when using parton densities with a more regular behaviour at small $x$, the hump should be more pronounced. This is in fact the case, as shown in fig. 2, obtained using the parton densities HMRS B化 15

The size of the hump relative to the total cross section can be taken as a measure of the importance of the small- $x$ regime. In fig. 3 we plot the contribution to the total cross section coming from the hump, relative to the total and to the $\mathcal{O}\left(\alpha_{S}^{2} \alpha_{\text {em }}\right)$ contribution. The hump contribution is defined as the integral of the $\mathcal{O}\left(\alpha_{S}^{2} \alpha_{\mathrm{em}}\right)$ term in $d \sigma / d \tau$, from its minimum point up to $\tau=1$. We are thus led to conclude that resummation effects could be important at the typical center-of-mass energies explored by HERA. Furthermore, flatter parton densities lead to larger small- $x$ effects. A similar pattern was found in the context of hadroproduction of bottom quarks, in ref. [16], where the all-order resummation of small- $x$ effects was actually performed for $b$ production at the Tevatron. Lacking a calculation of the effects of resummation in

\footnotetext{
${ }^{4}$ In this case the parton density is frozen to its value at the minimum $Q^{2}$ allowed by the parametrization.
} 


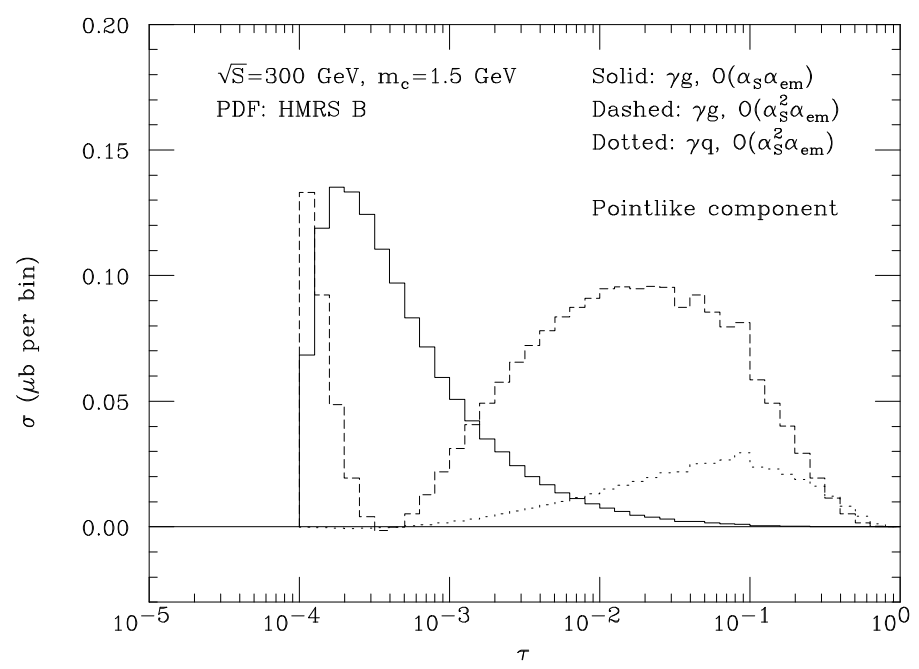

Figure 2: As in fig. 1, using HMRS B parton densities.

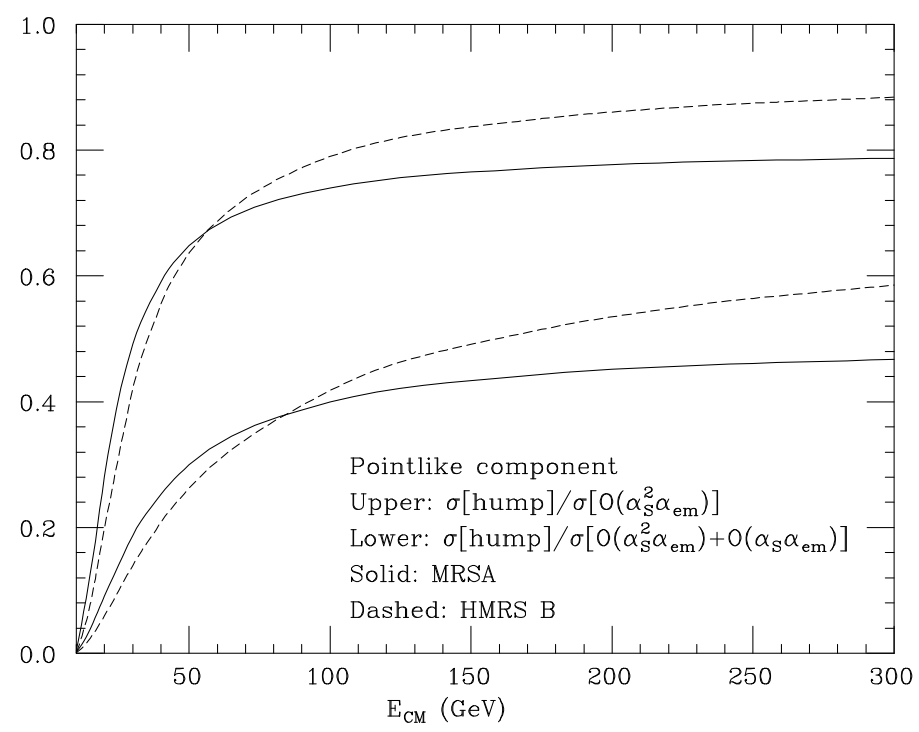

Figure 3: The contribution of the hump to the total cross section relative to the $\mathcal{O}\left(\alpha_{S}^{2} \alpha_{\mathrm{em}}\right)$ contribution (upper) and to the total (lower) for the MRSA and HMRS B proton parton densities. 
photoproduction, we will simply assume that it may give an enhancement equal to the square of the size of the hump relative to the total cross section, a pattern suggested by the results of ref. [16]. From fig. 3 we can then conclude that our calculation may underestimate the total charm cross section by $20 \%$ to $40 \%$, depending upon the parton density choice.

Sizeable small- $x$ effects are also present in the hadronic component of the charm cross section. In this case, we find that the relative importance of the hump contribution varies between $20 \%$ and $45 \%$ when the center-of-mass energy changes from 50 to $300 \mathrm{GeV}$ (this result was obtained using the MRSA and GRV-HO 17 parton densities in the proton and the photon respectively). The corresponding uncertainty on the final result is considerably smaller than other uncertainties.

Other important inputs in the computation of the total cross section are the value of $\Lambda_{5}^{\overline{\mathrm{MS}}}$ and the gluon density in the proton. The world average for $\alpha_{S}\left(M_{Z}^{2}\right)$ taken from the Particle Data Book, ref. [18], is $\alpha_{S}\left(M_{Z}^{2}\right)=0.117 \pm 0.005$, corresponding to $\Lambda_{5}^{\overline{\mathrm{MS}}}=215+65-55 \mathrm{MeV}$ (the value of $\Lambda_{5}^{\overline{\mathrm{MS}}}$ quoted in ref. 18 differs from ours because it actually refers to the 3-loop formula for $\alpha_{S}$, while we are quoting the 2loop value). On the other hand, the preferred value of $\Lambda_{5}^{\overline{\mathrm{MS}}}$ used in the latest parton density parametrizations of the CTEQ group is $158 \mathrm{MeV}$ (see ref. [19]), while the MRSA parametrization uses $151 \mathrm{MeV}$ (see ref. [14]). These values are below the lowest extreme of the world average range. This is due to the well known fact that the determinations of $\alpha_{S}$ from $e^{+} e^{-}$data tend to be higher than those from deepinelastic scattering. In order to assess the error in our predictions coming from the uncertainties on $\Lambda_{5}^{\overline{\mathrm{MS}}}$, we will therefore consider also the parton density set CTEQ2ML, which was fitted with $\Lambda_{5}^{\overline{\mathrm{MS}}}=220 \mathrm{MeV}$, a value close to the world average. In summary, our range for $\Lambda_{5}^{\overline{\mathrm{MS}}}$ is $151 \mathrm{MeV}<\Lambda_{5}^{\overline{\mathrm{MS}}}<220 \mathrm{MeV}$, which does not cover the full uncertainty on the present knowledge of $\alpha_{S}$. We are forced to do so, since larger values of $\Lambda_{5}^{\overline{\mathrm{MS}}}$ are too far from deep inelastic scattering determinations, and it would be difficult to assess their correlation with the gluon density in the proton.

Several uncertainties come into play in the extraction of the gluon density. Its normalization is strongly constrained by the momentum sum rule, while its shape, at relatively moderate value of $x$, is constrained by direct photon production data. Deep inelastic scattering data constrain the gluon density for relatively small values of $x$, where it affects the evolution of the singlet parton density. At very small 
values of $x$, the gluon density is in fact not yet known with precision. Both the CTEQ and MRS groups assume that its small- $x$ behaviour is the same as that of $F_{2}$, which is measured at HERA down to values of $x$ of the order of $10^{-4}$. On the other hand, a measurement of the gluon density at $Q^{2}=7 \mathrm{GeV}^{2}$ has recently been

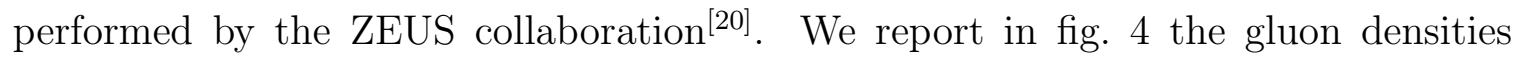
given by the sets MRSA, MRSD-' and CTEQ2MF, together with the two extreme small- $x$ parametrizations obtained by the ZEUS collaboration. The sets MRSD ${ }^{\prime}$ and

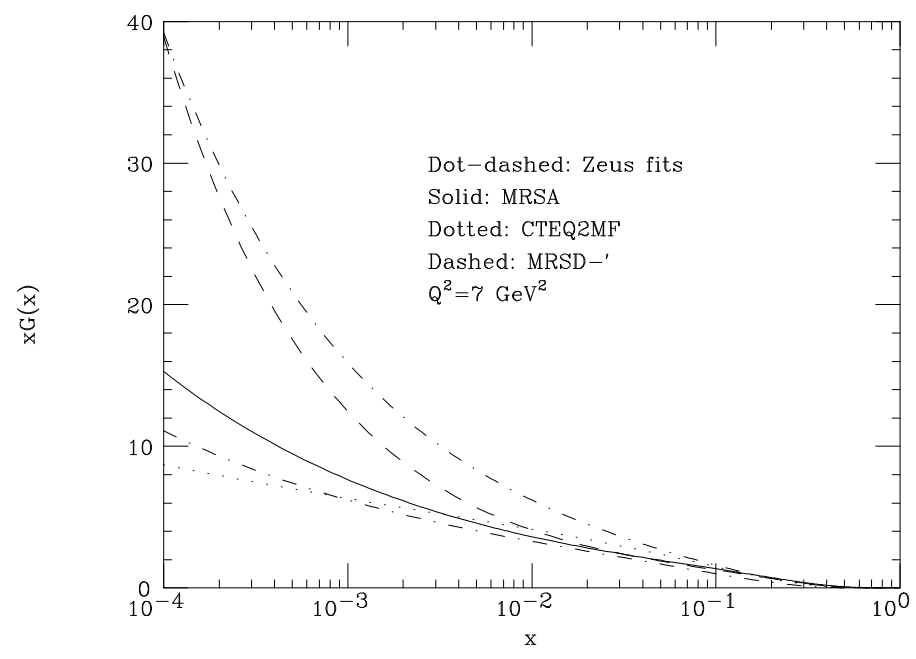

Figure 4: The gluon densities in MRSA, MRSD' and CTEQ2MF, together with the upper and lower parametrization of the ZEUS measurement.

CTEQ2MF differ essentially in the assumed small- $x$ behaviour. They are consistent with the two extreme parametrizations of the ZEUS data, and we will therefore use them as the two extreme possibilities.

Our results for the pointlike component of the charm cross section are shown in table 1 and in figs. 5 and 6. The strong sensitivity of the cross section to the charm quark mass is quite apparent. Scale sensitivity is also important, giving an uncertainty of a factor of 2 for the low mass value. From fig. 6 we also see that by comparing the cross section measured at HERA with lower energy measurements it should be possible to distinguish between different small- $x$ behaviour of the structure functions.

The hadronic component of the charm cross section is sensitive to the behaviour 


\begin{tabular}{|c|c|c|c|c|c|c|c|c|c|}
\hline & \multicolumn{3}{|c|}{$m_{c}=1.2 \mathrm{GeV}$} & \multicolumn{3}{|c|}{$m_{c}=1.5 \mathrm{GeV}$} & \multicolumn{3}{|c|}{$m_{c}=1.8 \mathrm{GeV}$} \\
\hline$E_{\mathrm{CM}} \quad \mu_{R}$ & $m_{c} / 2$ & $m_{c}$ & $2 m_{c}$ & $m_{c} / 2$ & $m_{c}$ & $2 m_{c}$ & $m_{c} / 2$ & $m_{c}$ & $2 m_{c}$ \\
\hline \multicolumn{10}{|c|}{ Proton PDF set MRSA } \\
\hline $30 \mathrm{GeV}$ & 2.582 & 2.104 & 1.712 & 1.321 & 1.090 & 0.907 & 0.735 & 0.611 & 0.515 \\
\hline $100 \mathrm{GeV}$ & 6.558 & 5.056 & 4.057 & 3.415 & 2.826 & 2.356 & 2.009 & 1.721 & 1.467 \\
\hline $280 \mathrm{GeV}$ & 13.89 & 10.13 & 8.008 & 7.314 & 5.858 & 4.831 & 4.389 & 3.685 & 3.120 \\
\hline \multicolumn{10}{|c|}{ Proton PDF set CTEQ2MF } \\
\hline $30 \mathrm{GeV}$ & 2.651 & 2.264 & 1.885 & 1.455 & 1.216 & 1.023 & 0.827 & 0.688 & 0.583 \\
\hline $100 \mathrm{GeV}$ & 4.371 & 3.856 & 3.236 & 2.699 & 2.425 & 2.088 & 1.761 & 1.593 & 1.393 \\
\hline $280 \mathrm{GeV}$ & 7.292 & 5.681 & 4.607 & 4.504 & 3.827 & 3.237 & 3.035 & 2.676 & 2.317 \\
\hline \multicolumn{10}{|c|}{ Proton PDF set MRSD-' } \\
\hline $30 \mathrm{GeV}$ & 3.136 & 2.363 & 1.885 & 1.422 & 1.136 & 0.936 & 0.748 & 0.612 & 0.513 \\
\hline $100 \mathrm{GeV}$ & 11.75 & 8.149 & 6.347 & 5.106 & 3.951 & 3.220 & 2.690 & 2.199 & 1.843 \\
\hline $280 \mathrm{GeV}$ & 34.16 & 23.06 & 17.81 & 14.79 & 11.17 & 9.033 & 7.794 & 6.247 & 5.196 \\
\hline \multicolumn{10}{|c|}{ Proton PDF set CTEQ2M } \\
\hline $30 \mathrm{GeV}$ & 2.696 & 2.182 & 1.785 & 1.341 & 1.107 & 0.926 & 0.734 & 0.612 & 0.519 \\
\hline $100 \mathrm{GeV}$ & 6.618 & 5.240 & 4.262 & 3.473 & 2.917 & 2.454 & 2.051 & 1.773 & 1.522 \\
\hline $280 \mathrm{GeV}$ & 13.46 & 10.17 & 8.157 & 7.207 & 5.898 & 4.919 & 4.374 & 3.728 & 3.184 \\
\hline \multicolumn{10}{|c|}{ Proton PDF set CTEQ2ML } \\
\hline $30 \mathrm{GeV}$ & 3.500 & 2.435 & 1.829 & 1.705 & 1.242 & 0.974 & 0.910 & 0.688 & 0.553 \\
\hline $100 \mathrm{GeV}$ & 8.818 & 5.625 & 4.171 & 4.270 & 3.169 & 2.497 & 2.451 & 1.942 & 1.583 \\
\hline $280 \mathrm{GeV}$ & 18.90 & 10.52 & 7.618 & 8.844 & 6.205 & 4.823 & 5.145 & 3.972 & 3.213 \\
\hline
\end{tabular}

Table 1: Pointlike component of the total charm cross sections ( $\mu b)$ in $\gamma p$ collisions, for various input parameters, as indicated. 


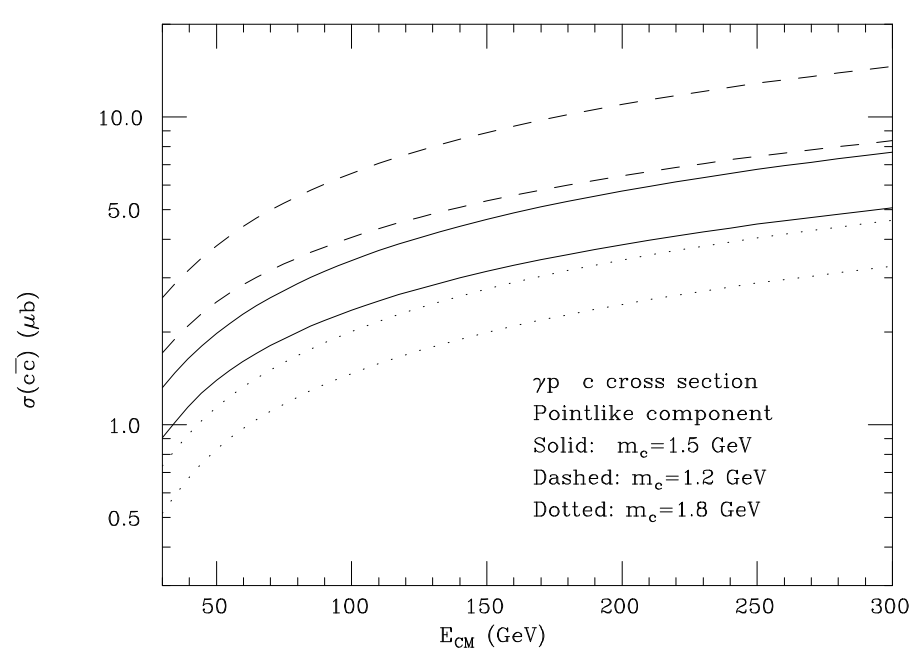

Figure 5: Pointlike component of the total charm cross section versus the center-of-mass energy in $\gamma p$ collisions. The bands are obtained by varying the renormalization scale between $m_{c} / 2$ and $2 m_{c}$. The proton parton density set is MRSA.

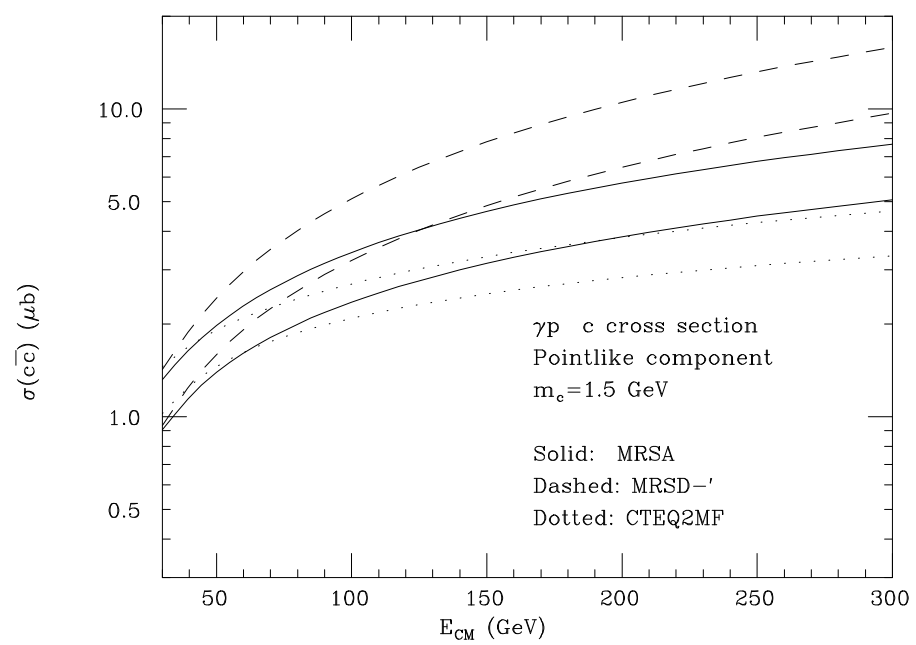

Figure 6: Pointlike component of the total charm cross section versus the center-of-mass energy in $\gamma p$ collisions for the three sets of parton densities $M R S A, M R S \perp^{-}$and CTEQ2MF. The bands are obtained by varying the renormalization scale between $m_{c} / 2$ and $2 m_{c}$. 
of the gluon density in the photon at relatively small values of $x$, a region not yet well explored experimentally. In fig. 7 we show the hadronic component cross section histogrammed as a function of $x_{\gamma}$ (the partonic $x$ in the photon). We see that the

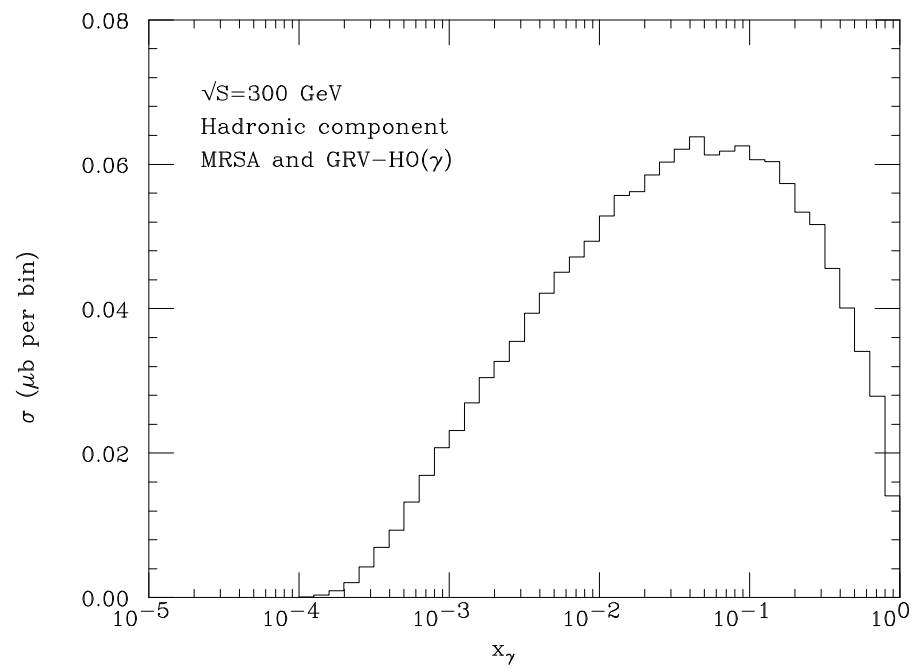

Figure 7: The $x_{\gamma}$ distribution of the hadronic component for charm production in $\gamma p$ collisions.

dominant values of $x_{\gamma}$ are between $10^{-2}$ and $10^{-1}$. Results from jet photoproduction at HERA 21 give some indication on the gluon density in the photon, whose extraction depends upon the value of the quark densities in the photon in a region where they are not directly probed. These data, in spite of the uncertainties, seem to indicate that the gluon density is between the one in the GRV-HO set [17] and the LAC1 set 22]. We note that the available experimental information on the gluon density in the photon do not allow to distinguish among different next-to-leading order parametrizations [23] of the parton distributions. Furthermore, the gluon density in the more recent leading order parametrization of ref. [24] is within the range spanned by the GRV-HO and the LAC1 sets. We will therefore take the sets GRV-HO and LAC1 as the two extremes. For the proton densities we will use the MRSA set. The relevant results are reported in table 2 and in fig. 8. In fig. 8 we plot the cross section without hadronic component, and with the hadronic component evaluated with the GRV-HO and LAC1 sets of photon parton densities. We conclude that with the present uncertainty it is quite possible that the hadronic component of the photon dominates the cross section 
at typical HERA energies. The pointlike and hadronic components of the cross section may be separated experimentally, at least in a first approximation, since in the case of the hadronic component a sizeable fraction of the photon momentum is lost into hadronic fragments. Therefore, at HERA it may be possible to distinguish between the two sets of parton densities in the photon we have considered.

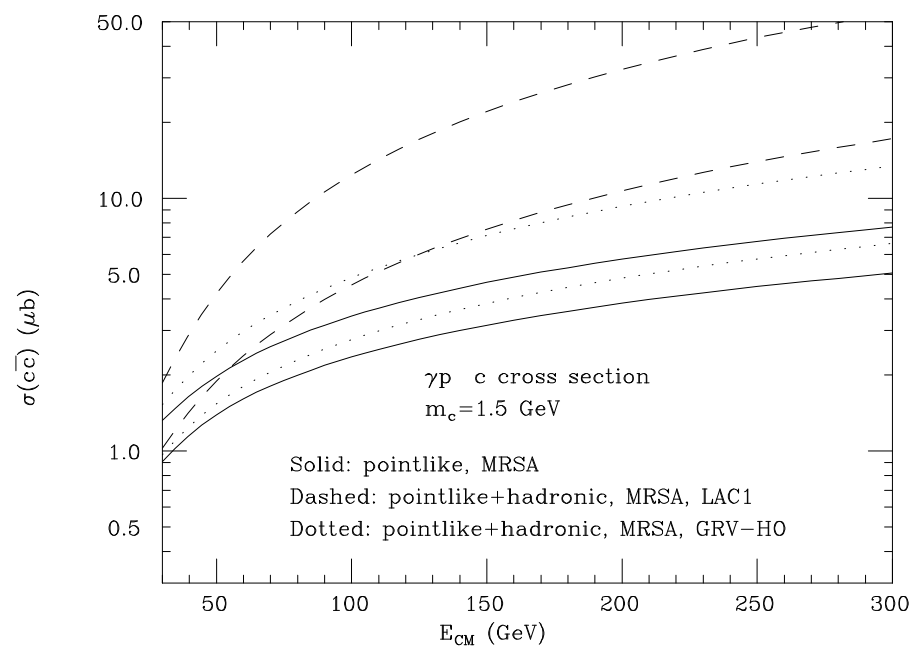

Figure 8: Total charm cross section versus the center-of-mass energy in $\gamma p$ collisions, with and without the inclusion of the hadronic component, computed with the two sets of photon parton densities LAC1 and GRV-HO. The bands are obtained by varying the renormalization scale between $m_{c} / 2$ and $2 m_{c}$.

\section{Bottom production}

In the case of bottom production, all the uncertainties we have discussed for charm are strongly reduced. The small- $x$ problem is much less dramatic. We have performed a study of the bottom cross section versus $\tau$, analogous to the one presented for charm in the previous section, and found that the size of the hump contribution is of the order of $20 \%$ ot the total cross section. The sensitivity of the pointlike cross section to the other parameters is given in table 3. Our central values of the cross section, in the column marked with DEF, are obtained with the renormalization and factorization scales set equal to $m_{b}$, and $m_{b}=4.75 \mathrm{GeV}$. If we vary all the 


\begin{tabular}{|l||c|c|c|c|c|c|c|c|c|c|}
\hline \multicolumn{1}{|c||}{} & \multicolumn{3}{c|}{$m_{c}=1.2 \mathrm{GeV}$} & \multicolumn{3}{c|}{$m_{c}=1.5 \mathrm{GeV}$} & \multicolumn{3}{c|}{$m_{c}=1.8 \mathrm{GeV}$} \\
\hline$E_{\mathrm{CM}} \quad \mu_{R}$ & $m_{c} / 2$ & $m_{c}$ & $2 m_{c}$ & $m_{c} / 2$ & $m_{c}$ & $2 m_{c}$ & $m_{c} / 2$ & $m_{c}$ & $2 m_{c}$ \\
\hline \hline \multicolumn{10}{|c|}{ Photon PDF set GRV-HO } \\
\hline $30 \mathrm{GeV}$ & 0.793 & 0.297 & 0.165 & 0.206 & 0.099 & 0.060 & 0.072 & 0.040 & 0.026 \\
\hline $100 \mathrm{GeV}$ & 4.572 & 1.680 & 0.924 & 1.438 & 0.673 & 0.405 & 0.589 & 0.314 & 0.200 \\
\hline $280 \mathrm{GeV}$ & 15.42 & 5.492 & 2.989 & 5.296 & 2.435 & 1.454 & 2.348 & 1.239 & 0.784 \\
\hline \hline \multicolumn{10}{|c|}{ Photon PDF set LAC1 } \\
\hline $30 \mathrm{GeV}$ & 3.350 & 0.995 & 0.503 & 0.540 & 0.213 & 0.119 & 0.125 & 0.059 & 0.036 \\
\hline $100 \mathrm{GeV}$ & 35.05 & 11.69 & 6.207 & 8.975 & 3.794 & 2.187 & 3.052 & 1.469 & 0.893 \\
\hline $280 \mathrm{GeV}$ & 140.3 & 48.77 & 26.30 & 42.45 & 18.84 & 11.09 & 16.78 & 8.494 & 5.283 \\
\hline
\end{tabular}

Table 2: Hadronic component of the total charm cross sections ( $\mu b)$ in $\gamma p$ collisions, for various input parameters, as indicated. The proton parton density set is MRSA.

parameters together in the direction that makes the cross section larger or smaller we get a spread for the bottom cross section which goes from $16 n \mathrm{~b}$ to $35 \mathrm{nb}$ at a center of mass energy of $100 \mathrm{GeV}$, and from $41 \mathrm{nb}$ to $101 \mathrm{nb}$ at $280 \mathrm{GeV}$. The results for the hadronic component are collected in table 1 . The default values correspond to $\mu_{R}=\mu_{F}=\mu_{\gamma}=m_{b}=4.75 \mathrm{GeV}$. We see that in this case the effect of the hadronic component is less dramatic. According to the LAC1 set, the hadronic contribution could be a substantial fraction of the total, while the GRV-HO set gives a much smaller result.

\section{Electroproduction of heavy quarks}

We present here our predictions for total charm and bottom cross sections in $e p$ collisions. The total electroproduction cross section in the Weizsäcker-Williams approximation is given by

$$
\sigma_{e p}(S)=\int d x f_{\gamma}^{(e)}\left(x, \mu_{W W}\right) \sigma_{\gamma p}(x S)
$$




\begin{tabular}{|c|c|c|c|c|c|c|c|}
\hline & & \multicolumn{2}{|c|}{$\mu_{R}$} & \multicolumn{2}{|c|}{$\mu_{F}$} & \multicolumn{2}{|c|}{$m_{b}(\mathrm{GeV})$} \\
\hline$E_{\mathrm{CM}}$ & DEF & $m_{b} / 2$ & $2 m_{b}$ & $m_{b} / 2$ & $2 m_{b}$ & 4.5 & 5 \\
\hline \multicolumn{8}{|c|}{ Proton PDF set MRSA } \\
\hline $100 \mathrm{GeV}$ & 22.90 & 26.00 & 20.36 & 21.87 & 23.63 & 27.41 & 19.28 \\
\hline $280 \mathrm{GeV}$ & 64.30 & 75.44 & 56.09 & 57.81 & 66.95 & 75.31 & 55.32 \\
\hline \multicolumn{8}{|c|}{ Proton PDF set CTEQ2MF } \\
\hline $100 \mathrm{GeV}$ & 24.91 & 27.93 & 22.33 & 23.80 & 25.73 & 29.70 & 21.02 \\
\hline $280 \mathrm{GeV}$ & 62.91 & 73.08 & 55.24 & 56.27 & 65.80 & 72.76 & 54.71 \\
\hline \multicolumn{8}{|c|}{ Proton PDF set MRSD- ${ }^{\prime}$} \\
\hline $100 \mathrm{GeV}$ & 23.03 & 26.19 & 20.45 & 22.18 & 23.63 & 27.75 & 19.26 \\
\hline $280 \mathrm{GeV}$ & 74.76 & 87.24 & 65.43 & 69.22 & 76.93 & 88.76 & 63.52 \\
\hline \multicolumn{8}{|c|}{ Proton PDF set CTEQ2M } \\
\hline $100 \mathrm{GeV}$ & 22.96 & 25.92 & 20.50 & 22.04 & 23.54 & 27.50 & 19.31 \\
\hline $280 \mathrm{GeV}$ & 65.33 & 75.96 & 57.32 & 59.43 & 67.51 & 76.40 & 56.23 \\
\hline \multicolumn{8}{|c|}{ Proton PDF set CTEQ2ML } \\
\hline $100 \mathrm{GeV}$ & 25.07 & 29.53 & 21.76 & 23.38 & 25.92 & 30.03 & 21.07 \\
\hline $280 \mathrm{GeV}$ & 71.04 & 86.84 & 60.42 & 61.74 & 74.39 & 82.99 & 61.20 \\
\hline
\end{tabular}

Table 3: Pointlike component of the total bottom cross sections ( $n b$ ) in $\gamma p$ collisions, for various input parameters, as indicated. 


\begin{tabular}{|l||l||l|l||l|l||l|l||c|c|}
\hline \multicolumn{1}{|c||}{} & \multicolumn{1}{c||}{$\mu_{R}$} & \multicolumn{2}{c||}{$\mu_{F}$} & \multicolumn{3}{c||}{$\mu_{\gamma}$} & \multicolumn{2}{c|}{$m_{b}(\mathrm{GeV})$} \\
\hline$E_{\mathrm{CM}}$ & DEF & $m_{b} / 2$ & $2 m_{b}$ & $m_{b} / 2$ & $2 m_{b}$ & $m_{b} / 2$ & $2 m_{b}$ & 4.5 & 5 \\
\hline \hline \multicolumn{10}{|c||}{ Photon PDF set GRV-HO } \\
\hline $100 \mathrm{GeV}$ & 4.244 & 5.589 & 3.290 & 4.246 & 4.258 & 3.883 & 4.525 & 5.444 & 3.347 \\
\hline $280 \mathrm{GeV}$ & 27.63 & 38.67 & 20.74 & 25.68 & 29.13 & 25.39 & 28.68 & 34.29 & 22.48 \\
\hline \hline \multicolumn{10}{|c|}{ Photon PDF set LAC1 } \\
\hline $100 \mathrm{GeV}$ & 6.693 & 9.264 & 5.053 & 7.157 & 6.394 & 7.161 & 6.348 & 9.309 & 4.890 \\
\hline $280 \mathrm{GeV}$ & 98.45 & 137.2 & 74.05 & 96.07 & 100.6 & 106.4 & 92.18 & 128.7 & 76.15 \\
\hline
\end{tabular}

Table 4: Hadronic component of the total bottom cross sections ( $n$ b) in $\gamma p$ collisions, for various input parameters, as indicated. The proton parton density set is MRSA.

In the following, unless otherwise specified, we will refer to the case when no anti-tag condition is imposed on the outgoing electron. The function $f_{\gamma}^{(e)}$ is given in this case by

$$
f_{\gamma}^{(e)}\left(x, \mu_{W W}\right)=\frac{\alpha_{\mathrm{em}}}{2 \pi} \frac{1+(1-x)^{2}}{x} \log \frac{\mu_{W W}^{2}(1-x)}{m_{e}^{2} x^{2}} .
$$

The scale $\mu_{W W}$, according to ref. [25], will be taken equal to the quark mass for the pointlike component.

The choice of $\mu_{W w}$ in the case of the hadronic component is a debatable issue. Intuitive reasoning leads to conclude that this scale should not be larger than a typical hadronic scale, because a strongly off-shell photon would have smaller probability of mixing with hadronic resonances. Some authors (see refs. [26,27,28]) suggest instead that this scale should be related to the hardness of the process, and should therefore be larger for bottom production than for charm production. In practice, in the charm case, changing $\mu_{W W}$ from $1 \mathrm{GeV}$ up to the quark mass has only a few percent effect on the size of the hadronic component for charm production, while for bottom production the effect could be as large as $20 \%$. In both cases, as we will see, these effects are, at present, much smaller than other sources of uncertainty. In the following we will choose $\mu_{W W}=1 \mathrm{GeV}$ for charm and $\mu_{W W}=3 \mathrm{GeV}$ for bottom. We also notice that, in the case when an anti-tagging condition is imposed, this problem is not relevant 


\begin{tabular}{|l||c|c|c|c|c|c|c|c|c|}
\hline \multicolumn{1}{|c||}{} & \multicolumn{3}{c|}{$m_{c}=1.2 \mathrm{GeV}$} & \multicolumn{3}{c|}{$m_{c}=1.5 \mathrm{GeV}$} & \multicolumn{3}{c|}{$m_{c}=1.8 \mathrm{GeV}$} \\
\hline \multicolumn{1}{|c||}{$\mu_{R}$} & $m_{c} / 2$ & $m_{c}$ & $2 m_{c}$ & $m_{c} / 2$ & $m_{c}$ & $2 m_{c}$ & $m_{c} / 2$ & $m_{c}$ & $2 m_{c}$ \\
\hline \hline MRSA & 1.478 & 1.113 & 0.888 & 0.749 & 0.605 & 0.500 & 0.431 & 0.361 & 0.305 \\
\hline CTEQ2MF $^{\prime}$ & 1.130 & 0.923 & 0.761 & 0.630 & 0.535 & 0.453 & 0.385 & 0.334 & 0.288 \\
\hline MRSD- $^{\prime}$ & 2.472 & 1.713 & 1.333 & 1.087 & 0.833 & 0.677 & 0.574 & 0.463 & 0.387 \\
\hline CTEQ2ML & 2.078 & 1.250 & 0.918 & 0.956 & 0.678 & 0.529 & 0.530 & 0.406 & 0.327 \\
\hline
\end{tabular}

Table 5: Pointlike component of the total charm cross sections $(\mu b)$ in ep collisions, at $E_{C M}=314 \mathrm{GeV}$, for various input parameters, as indicated.

at all.

We present in tables 5 and 6 our predictions for the total charm cross sections, for the pointlike and the hadronic component respectively, at $E_{C M}=314 \mathrm{GeV}$. The proton and photon parton densities are chosen as in the case of photoproduction. We do not consider here the CTEQ2M set, which gives essentially the same results as our default set MRSA. We observe that the most important source of uncertainty is the charm mass. The ratio bewteen the extreme choices $m_{c}=1.2$ and $1.8 \mathrm{GeV}$ can be as large as 4.3 for the pointlike component and 10 for the hadronic component, and becomes larger for smaller values of the renormalization scale. The renormalization scale variation has a strong effect on the hadronic component (a factor up to 5.5 for the lowest value of the charm mass), and quite a sizeable effect on the pointlike component. As in the photoproduction case, the differences in the hadronic component evaluated with GRV-HO and LAC1 densities are striking. For our central values of $m_{c}=1.5 \mathrm{GeV}$ and $\mu_{R}=m_{c}$, the hadronic contribution obtained with the GRV-HO set is $20 \%$ of the pointlike component, while the hadronic and pointlike contributions are comparable when using the LAC1 set.

We also calculated charm electroproduction cross sections with the anti-tagging condition imposed, as in ref. [10]; the results can be obtained from those presented in tables 5 and 6 by multiplying by a factor of 0.7 , which is approximately independent of the parameters of the calculation.

We have checked that varying the factorization scale of the photon in the pointlike component has a very small effect on the results. 


\begin{tabular}{|l||c|c|c|c|c|c|c|c|c|}
\hline \multicolumn{1}{|c||}{} & \multicolumn{3}{c|}{$m_{c}=1.2 \mathrm{GeV}$} & \multicolumn{3}{c|}{$m_{c}=1.5 \mathrm{GeV}$} & \multicolumn{3}{c|}{$m_{c}=1.8 \mathrm{GeV}$} \\
\hline \multicolumn{1}{|c||}{$\mu_{R}$} & $m_{c} / 2$ & $m_{c}$ & $2 m_{c}$ & $m_{c} / 2$ & $m_{c}$ & $2 m_{c}$ & $m_{c} / 2$ & $m_{c}$ & $2 m_{c}$ \\
\hline \hline GRV-HO & 0.878 & 0.320 & 0.176 & 0.277 & 0.129 & 0.078 & 0.115 & 0.061 & 0.039 \\
\hline LAC1 & 6.697 & 2.258 & 1.204 & 1.796 & 0.774 & 0.450 & 0.646 & 0.319 & 0.196 \\
\hline
\end{tabular}

Table 6: Hadronic component of the total charm cross sections $(\mu b)$ in ep collisions, at $E_{C M}=314 \mathrm{GeV}$, for various input parameters, as indicated. The MRSA set for the densities in the proton was used.

In our analyses, we have kept the factorization scales $\mu_{F}$ and $\mu_{\gamma}$ fixed, since parton densities are usually available only for momentum scales above $\sim 5 \mathrm{GeV}^{2}$. There are actually two independent next-to-leading order parametrizations of densities in the proton valid for $Q^{2}<m_{c}^{2}$ : an extension of the MRSA set [29], which we will call MRSA modified, and the GRV-HO set [30]. We have used these two parametrizations to perform a study of the factorization scale dependence of the charm cross section.

The MRSA modified set is obtained by ordinary Altarelli-Parisi evolution of the MRSA set, supplemented with some specific assumptions on non-perturbative effects. On the other hand, the GRV-HO set has no non-perturbative assumptions. It is obtained by perturbative evolution, starting from "valence-like" inputs at $Q_{0}^{2}=0.3$ $\mathrm{GeV}^{2}$. No attempt is made to mimic the low- $Q^{2}$ data. The parametrization of nonperturbative effects inserted in the MRSA set is, strictly speaking, in contrast with the prescriptions of the factorization theorem. Nevertheless, we verified that these phenomenologically motivated inputs are numerically not important for factorization scales around the charm mass and larger. As far as the GRV set is concerned, the validity of the Altarelli-Parisi evolution equations and the perturbative expansion at the very low momentum scale at which the evolution is started is questionable. However, we believe that both sets are well suited for the purpose of estimating the uncertainties arising from the factorization scale variation.

In fig. 9 we plot the ratio

$$
R=\frac{\sigma\left(\mu=\xi m_{c}\right)}{\sigma\left(\mu=2 m_{c}\right)}
$$

as a function of $\xi$. The dot-dashed curve is obtained with $\mu_{R}=\mu$, and keeping 


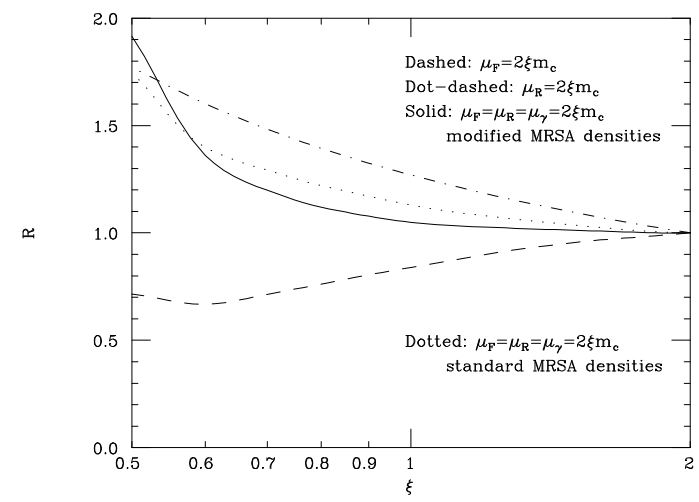

(a)

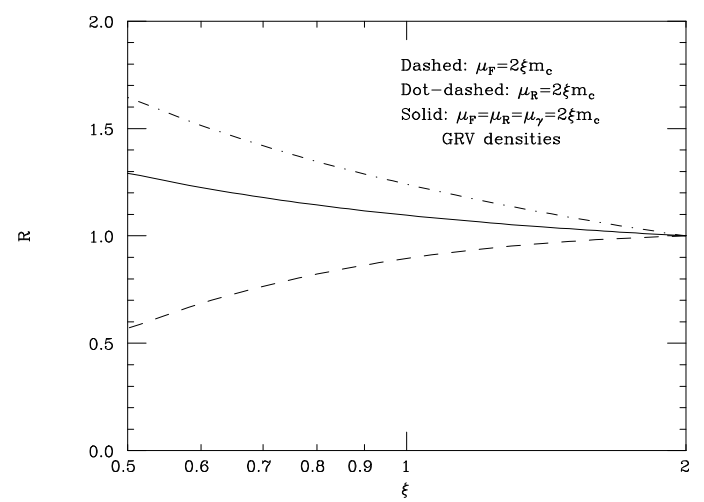

(b)

Figure 9: The ratio $R$, defined in the text, for different choices of factorization and renormalization scales. MRSA (a) and GRV (b) densities in the proton are used.

$\mu_{F}=\mu_{\gamma}=2 m_{c}$, the dashed curve has $\mu_{F}=\mu, \mu_{R}=m_{c}$ and $\mu_{\gamma}=2 m_{c}$, and the solid curve has $\mu_{F}=\mu_{R}=\mu_{\gamma}=\mu$. In the case of the MRSA parton densities, we also show (dotted curve) the same quantity, for $\mu_{F}=\mu_{R}=\mu_{\gamma}=\mu$, with the parametrization of non-perturbative effects removed. In eq. (4.3) $\sigma$ is the full (poinlike plus hadronic) cross section for an untagged electron and $m_{c}=1.5 \mathrm{GeV}$. We use the GRV-HO set for the densities in the photon. When varying the hadronic factorization scale, the cross section, in the case of the MRSA modified set, decreases approaching the very low momentum region, where it increases again. This effect is essentially due to targetmass corrections in the parton densities, which sizeably harden the densities at low scales, and which are not present in the GRV set. The solid line is the result for the simultaneous variation of all the scales involved, including the photon factorization scale. We stress therefore that in this case we are probing also photon densities at low momenta. From this study we conclude that variation of the factorization scale brings about an additional uncertainty of $40 \%$ in the direction of lower cross sections.

In tables $\square$ and 8 we present the pointlike and hadronic components of the total cross sections for bottom production at $E_{C M}=314 \mathrm{GeV}$. The defalut values correspond to all the scales equal to $m_{b}$, and $m_{b}=4.75 \mathrm{GeV}$. As expected, in this case the 


\begin{tabular}{|l||l||c|c||c|c||c|c|}
\hline \multicolumn{1}{|c||}{} & \multicolumn{1}{c||}{} & \multicolumn{2}{c||}{$\mu_{R}$} & \multicolumn{2}{c||}{$\mu_{F}$} & \multicolumn{2}{c|}{$m_{b}(\mathrm{GeV})$} \\
\hline & DEF & $m_{b} / 2$ & $2 m_{b}$ & $m_{b} / 2$ & $2 m_{b}$ & 4.5 & 5 \\
\hline \hline MRSA & 4.640 & 5.332 & 4.098 & 4.391 & 4.773 & 5.541 & 3.917 \\
\hline CTEQ2MF & 4.814 & 5.469 & 4.283 & 4.554 & 4.958 & 5.720 & 4.079 \\
\hline MRSD- & 4.856 & 5.580 & 4.288 & 4.649 & 4.963 & 5.839 & 4.073 \\
\hline CTEQ2ML & 5.123 & 6.110 & 4.418 & 4.728 & 5.290 & 6.113 & 4.325 \\
\hline
\end{tabular}

Table 7: Pointlike component of the total bottom cross sections ( $\mathrm{nb}$ ) in ep collisions, for various input parameters, as indicated, and $E_{\mathrm{CM}}=314 \mathrm{GeV}$.

\begin{tabular}{|l||c||c|c||c|c||c|c||c|c|}
\hline \multicolumn{1}{|c||}{} & \multicolumn{1}{|c||}{} & \multicolumn{2}{c||}{$\mu_{R}$} & \multicolumn{2}{c||}{$\mu_{F}$} & \multicolumn{3}{c||}{$\mu_{\gamma}$} & \multicolumn{2}{c|}{$m_{b}(\mathrm{GeV})$} \\
\hline & DEF & $m_{b} / 2$ & $2 m_{b}$ & $m_{b} / 2$ & $2 m_{b}$ & $m_{b} / 2$ & $2 m_{b}$ & 4.5 & 5 \\
\hline \hline GRV-HO & 1.115 & 1.510 & 0.851 & 1.077 & 1.146 & 1.018 & 1.177 & 1.408 & 0.892 \\
\hline LAC1 & 2.842 & 3.947 & 2.142 & 2.861 & 2.840 & 3.067 & 2.675 & 3.792 & 2.157 \\
\hline
\end{tabular}

Table 8: Hadronic component of the total bottom cross sections ( $n$ b) in ep collisions, for various input parameters, as indicated, and $E_{\mathrm{CM}}=314 \mathrm{GeV}$. The proton parton density set is MRSA.

sensitivity to scale variations is much smaller than in the charm case. The sensitivity to the choice of parton densities is even less important than that arising from scale variation. This is because the small- $x$ region, where the various sets mostly differ, is not deeply probed in the case of bottom production. The differences between results from various parton density sets come mainly from the different values of $\Lambda$.

The hadronic component, displayed in table 8 , is at most $75 \%$ of the pointlike component, even when the LAC1 set is used. Again, this is related to the fact that the small- $x$ region, where LAC1 gluon density is much larger than the GRV-HO one, is not so important in this case. In the case of bottom, electroproduction cross sections with the anti-tagging condition imposed on the scattered electron are approximately a factor 0.6 smaller than those presented in tables 0 and 8 , independently of all other parameters. 


\section{Conclusions}

Charm and bottom photoproduction cross sections are theoretically in a better shape than corresponding hadroproduction cross sections. We have presented results which indicate that the small- $x$ effects should be under control at HERA energies, even for charm production, and that a measurement of the pointlike component of the charm photoproduction cross section at HERA could help to discriminate among various possible small- $x$ behaviours of the gluon densities in the proton and in the photon.

We found that the most important source of uncertainty is coming from the hadronic component of the cross section. If the gluon density in the photon is as large as the LAC1 parametrization suggests, then the hadronic component will be comparable or larger than the pointlike component for energies above $100 \mathrm{GeV}$ for charm, and for energies of the order of $280 \mathrm{GeV}$ for bottom. Hopefully, by looking at the structure of the events, experiments may be able to separate the pointlike and hadronic components, and therefore assess their relative magnitude.

We also performed a study on total electroproduction cross sections in the Weizsäcker-Williams approximation, including recent parton density parametrizations, the effect of an anti-tagging condition on the electron, and a more thorough illustration of the sensitivity to the various physical parameters. We find results that are in substantial agreement with earlier studies (ref. [10]).

\section{Acknowledgements}

We would like to thank M. Fontannaz, K. Hagiwara, G. Iacobucci, R. Prosi, J. Roldan, A. Staiano, W. J. Stirling and M. Tanaka for providing us with useful information. 


\section{References}

[1] G. Bellini, proceedings of "Les Rencontres de Physique de la Vallee d'Aoste",

La Thuile, Aosta Valley, March 6-12, 1994;

J.C. Anjos et al., E691 Coll., Phys. Rev. Lett. 62(1989)513;

M. P. Alvarez et al., NA14/2 Coll., Z. Phys. C60(1993)53.

[2] S. Frixione, M. L. Mangano, P. Nason and G. Ridolfi, preprint CERN-TH.7292/94, GEF-TH-4/1994, to appear in Nuclear Physics B.

[3] H1 Coll., preprint DESY-94-187, 1994.

[4] C. Albajar et al., UA1 Coll., Phys. Lett. B256(1991)121.

[5] K. Byrum, CDF Coll., presented at the XXIV International Conference on High Energy Physics, Glasgow, Scotland, 20-27 July 1994, preprint FERMILABConf-94/325-E.

[6] D. Hedin, D0 Coll., presented at the XXIV International Conference on High Energy Physics, Glasgow, Scotland, 20-27 July 1994 preprint FERMILABConf-94/415.

[7] S. Riemersma, J. Smith and W. L. van Neerven, preprint SMU-HEP 94-25, ITP-SB-94-59, INLO-PUB-16/94, hep-ph/9411431

[8] R.K. Ellis and P. Nason, Nucl. Phys. B312(1989)551.

[9] P. Nason, S. Dawson and R. K. Ellis, Nucl. Phys. B303(1988)607; B327(1988)49.

[10] S. Frixione, M. L. Mangano, P. Nason and G. Ridolfi, Phys. Lett. B308(1993)137.

[11] M.L. Mangano, P. Nason and G. Ridolfi, Nucl. Phys. B373(1992)295.

[12] S. Frixione, M. L. Mangano, P. Nason and G. Ridolfi, Nucl. Phys. B412(1994)225.

[13] R.K. Ellis and D.A. Ross, Nucl. Phys. B345(1990)79;

S. Catani, M. Ciafaloni and F. Hautmann, Phys. Lett. B242(1990)97, Nucl. Phys. B366(1991)135, Nucl. Phys. B (Proc. Suppl.) 23B(1991)328.

[14] A. D. Martin, R. G. Roberts and W. J. Stirling, preprint RAL-94-055, DTP/94/34. 
[15] P. Harriman, A. Martin, R. Roberts and W.J. Stirling, Phys. Rev. D37(1990)798.

[16] J.C. Collins and R.K. Ellis, Nucl. Phys. B360(1991)3.

[17] M. Glück, E. Reya and A. Vogt, Phys. Rev. D46(1992)1973.

[18] Review of Particle Properties, Phys. Rev. D50 Part I, p. 1297.

[19] H.L. Lai et al., preprint MSU-HEP-41024, CTEQ-404, hep-ph-9410404.

[20] ZEUS Coll., preprint DESY-94-192, 1994.

[21] T. Haas, preprint DESY 94-160, Sept. 1994.

[22] H. Abramowicz, K. Charchula and A. Levy, Phys. Lett. 269B(1991)458.

[23] L. E. Gordon and J. K. Storrow, Z. Phys. C56(1992)307;

P. Aurenche, M. Fontannaz and J.-Ph. Guillet, preprint ENSLAPP-A-435-93, LPTHE Orsay 93-37.

[24] K. Hagiwara, M. Tanaka and I. Watanabe, preprint KEK-TH-376, hep-ph/9406252.

[25] S. Frixione, M. L. Mangano, P. Nason and G. Ridolfi, Phys. Lett. B319(1993)339.

[26] T. Uematsu and I. F. Walsh, Nucl. Phys. B199(1982)93.

[27] F. Borzumati and G. A. Schuler, Z. Phys. C58(1992)139.

[28] M. Drees and R. M. Godbole, Phys. Rev. D50(1994)3124.

[29] A. D. Martin, R. G. Roberts and W. J. Stirling, preprint RAL-94-104, DTP/94/78.

[30] M. Glück, E. Reya and A. Vogt, Z. Phys. C53(1992)127. 